\title{
Investigation of beauty production and parton shower effects at the LHC
}

\author{
H. Jung, ${ }^{1,2}$ M. Kraemer, ${ }^{1}$ A. V. Lipatov, ${ }^{3}$ and N. P. Zotov ${ }^{3}$ \\ ${ }^{1}$ DESY, Hamburg, Germany \\ ${ }^{2}$ CERN, Geneva, Switzerland University of Antwerp, Antwerp, Belgium \\ ${ }^{3}$ Skobeltsyn Institute of Nuclear Physics, Lomonosov Moscow State University, Russia
}

(Received 13 November 2011; published 22 February 2012)

\begin{abstract}
We present hadron-level predictions from the Monte Carlo generator CASCADE and parton-level calculations of open $b$ quark, $b$-flavored hadron, and inclusive $b$-jet production in the framework of the $k_{T}$-factorization QCD approach for the LHC energies. The unintegrated gluon densities in a proton are determined using the Catani-Ciafaloni-Fiorani-Marchesini (CCFM) evolution equation and the KimberMartin-Ryskin (KMR) prescription. Our predictions are compared with the first data taken by the CMS and LHCb collaborations at $\sqrt{s}=7 \mathrm{TeV}$. We study the theoretical uncertainties of our calculations and investigate the effects coming from parton showers in initial and final states. The special role of initial gluon transverse momenta in description of the data is pointed out.
\end{abstract}

DOI: 10.1103/PhysRevD.85.034035

PACS numbers: $12.38 .-\mathrm{t}, 13.85 .-\mathrm{t}$

\section{INTRODUCTION}

Beauty production at high energies is the subject of intense study from both theoretical and experimental points of view since events containing $b$ quarks present an important background to many of the searches at the LHC. From a theoretical point of view, the dominant production mechanism is believed to be quark-pair production through the gluon-gluon fusion subprocess. Therefore, these processes provide an opportunity to test the different predictions based on Quantum Chromodynamics (QCD). The present note is motivated by the recent measurements [1-6] of beauty production performed by the CMS and LHCb collaborations at the LHC energy $\sqrt{s}=7 \mathrm{TeV}$. It was observed [2-4] that the data on $B^{+}, B^{0}$-meson, and open $b$-quark production tend to be higher than the MC@NLO $[7,8]$ predictions. There are no predictions which describe reasonably well the angular correlations between $b$-flavored hadrons measured [5] by the CMS collaboration. On the other hand, the measurements of transverse momenta and rapidity distributions of beauty hadrons [1] and inclusive $b$-jets [6] are reasonably well-described by the MC@NLO.

In the framework of the $k_{T}$-factorization approach of QCD [9], heavy quark production has been studied (for previous results see [10-16]). In our previous paper, [16] we obtained good agreement between the Tevatron data on the open $b$ quarks, $b \bar{b}$ di-jets, $B^{+}$, and several $D$-mesons (or rather muons from their semileptonic decays) production with the predictions coming from $k_{T}$ factorization. We also investigated the role of initial- and final-state parton showers. We have shown that a good description of the specific angular correlations between the final-state particles is obtained in Monte Carlo event generator CASCADE [17] once the higher-order process $g g^{*} \rightarrow g g$ with subsequent $g \rightarrow b \bar{b}$ splitting is included, which is not discussed here.
Based on these results, here we give a systematic analysis of the recent CMS and $\mathrm{LHCb}$ data [1-6] on beauty production in the framework of $k_{T}$-factorization. ${ }^{1}$ Following [16], we produce the calculations in two ways: we perform numerical parton-level calculations (labeled as Lipatov-Zotov or LZ) as well as calculations with the full hadron-level Monte Carlo event generator CASCADE and compare both with the measured cross sections. ${ }^{2}$ We investigate the influence of parton showers in initial and final states for the description of LHC data. Specially, we concentrate on the angular correlations between the produced $b$-flavored hadrons measured by the CMS collaboration [5], which are important for our understanding of production dynamics [14-16]. Finally, we study the different sources of theoretical uncertainties, i.e. uncertainties connected with the gluon evolution scheme, heavy quark mass, hard scale of partonic subprocess, and the heavy quark fragmentation functions.

Our paper is organized as follows. In Sec. II, we recall the basic formulas of the $k_{T}$-factorization approach with a brief review of calculation steps. In Sec. III, we present the numerical results of our calculations and a discussion. Section IV contains our conclusions.

\section{THEORETICAL FRAMEWORK}

In this paper, we follow the approach described in earlier publications [14-16]. For the reader's convenience, we only briefly recall main points of the theoretical scheme. The cross section of heavy quark production in $p p$ collisions at high energies in the $k_{T}$-factorization approach is calculated as a convolution of the off shell (i.e. $k_{T}$-dependent) partonic cross section and the unintegrated

\footnotetext{
${ }^{1}$ See also [18].

${ }^{2}$ In addition to the comparison of CASCADE predictions with the data in [4], we present further studies here.
} 
gluon distributions in a proton. It can be presented in the following form:

$$
\begin{aligned}
\sigma(p p & \rightarrow Q \bar{Q} X) \\
= & \int \frac{1}{16 \pi\left(x_{1} x_{2} s\right)^{2}} \mathcal{A}\left(x_{1}, \mathbf{k}_{1 T}^{2}, \mu^{2}\right) \mathcal{A}\left(x_{2}, \mathbf{k}_{2 T}^{2}, \mu^{2}\right) \\
& \times\left|\overline{\mathcal{M}}\left(g^{*} g^{*} \rightarrow Q \bar{Q}\right)\right|^{2} \\
& \times d \mathbf{p}_{1 T}^{2} d \mathbf{k}_{1 T}^{2} d \mathbf{k}_{2 T}^{2} d y_{1} d y_{2} \frac{d \phi_{1}}{2 \pi} \frac{d \phi_{2}}{2 \pi},
\end{aligned}
$$

where $\mathcal{A}\left(x, \mathbf{k}_{T}^{2}, \mu^{2}\right)$ is the unintegrated gluon distribution in a proton, $\left|\overline{\mathcal{M}}\left(g^{*} g^{*} \rightarrow Q \bar{Q}\right)\right|^{2}$ is the off shell (i.e. depending on the initial gluon virtualities $\mathbf{k}_{1 T}^{2}$ and $\mathbf{k}_{2 T}^{2}$ ) matrix element squared and averaged over initial gluon polarizations and colors, and $s$ is the total center-of-mass energy. The produced heavy quark $Q$ and antiquark $\bar{Q}$ have the transverse momenta $\mathbf{p}_{1 T}$ and $\mathbf{p}_{2 T}$ and the center-of-mass rapidities $y_{1}$ and $y_{2}$. The initial off shell gluons have fractions $x_{1}$ and $x_{2}$ of the parent protons' longitudinal momenta, nonzero transverse momenta $\mathbf{k}_{1 T}$ and $\mathbf{k}_{2 T}$ $\left(\mathbf{k}_{1 T}^{2}=-k_{1 T}^{2} \neq 0, \mathbf{k}_{2 T}^{2}=-k_{2 T}^{2} \neq 0\right)$, and azimuthal angles $\phi_{1}$ and $\phi_{2}$. The analytic expression for the $\left|\overline{\mathcal{M}}\left(g^{*} g^{*} \rightarrow Q \bar{Q}\right)\right|^{2}$ can be found, for example, in $[9,13]$.

The unintegrated gluon distributions in the proton $\mathcal{A}\left(x, \mathbf{k}_{T}^{2}, \mu^{2}\right)$ involved in Eq. (1) can be obtained from the analytical or numerical solutions of the Balitsky-FadinKuraev-Lipatov [19] or CCFM [20] evolution equations.
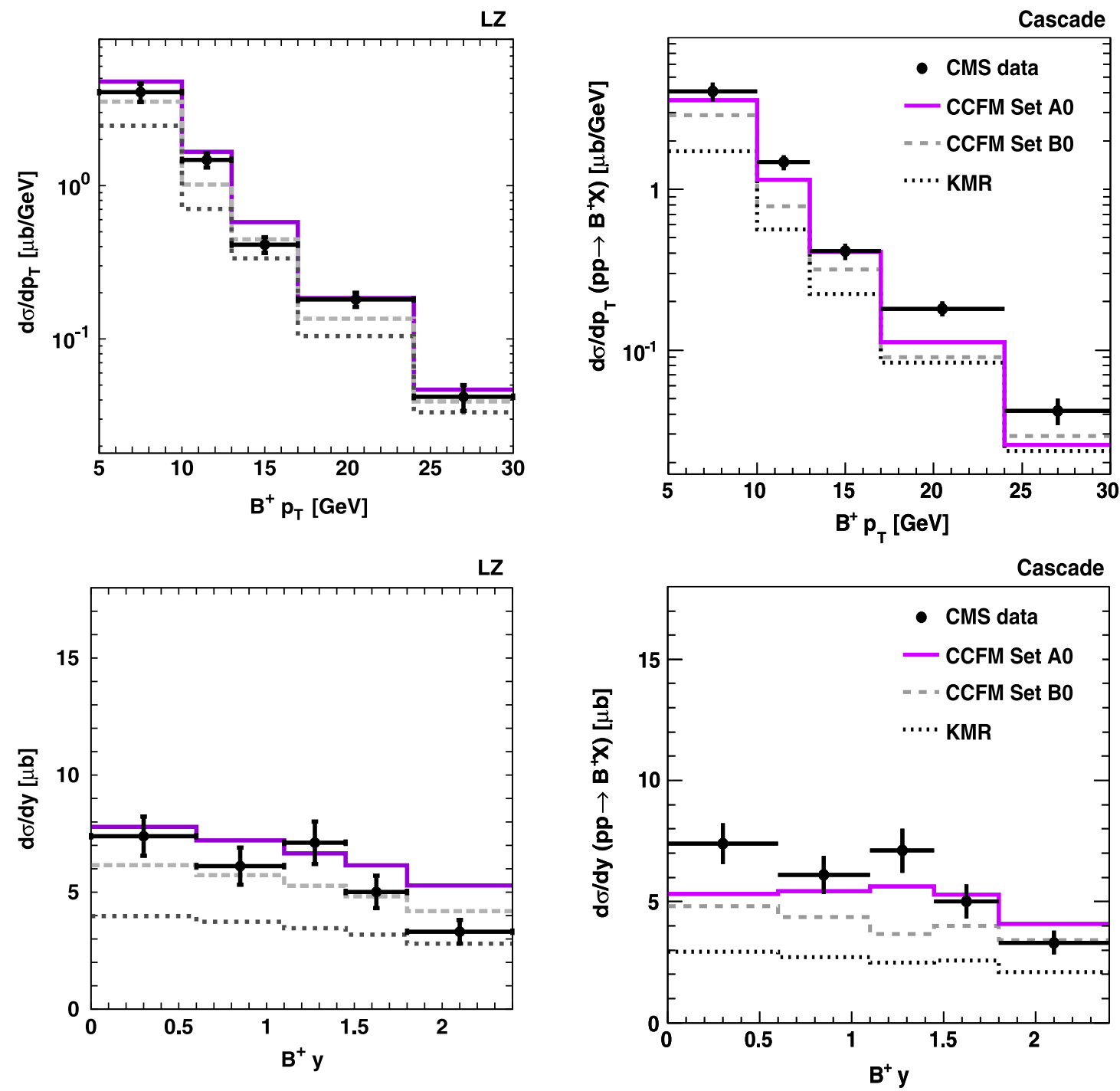

FIG. 1 (color online). The transverse-momentum and rapidity distributions of $B^{+}$-meson production at the LHC. The kinematical cuts applied are described in the text. The solid, dashed and dotted histograms correspond to the results obtained with the CCFM set A0, B0, and KMR unintegrated gluon densities. The first column shows the LZ results while the second one depicts the CASCADE predictions. The experimental data are from CMS [2]. 
As in [12], we have tested a few different sets. In the first of them, CCFM set A0 was obtained in [21] from the CCFM equation where all input parameters were fitted to describe the proton-structure function $F_{2}\left(x, Q^{2}\right)$. An equally good fit was obtained using different values for the soft cut and a different value for the width of the intrinsic $\mathbf{k}_{T}$ distribution (CCFM set B0). We also used the unintegrated gluon densities in a proton taken in the Kimber-Martin-Ryskin form [22]: The KMR approach is a formalism to construct the unintegrated parton distributions from well-known conventional ones. For the input, we used the standard Martin-Stirling-Thorne-Watt '2008 (LO)[23] in Lipatov-Zotov model (LZ) calculations and the Martin-Roberts-Stirling-Thorne '99[24] (in CASCADE) sets.
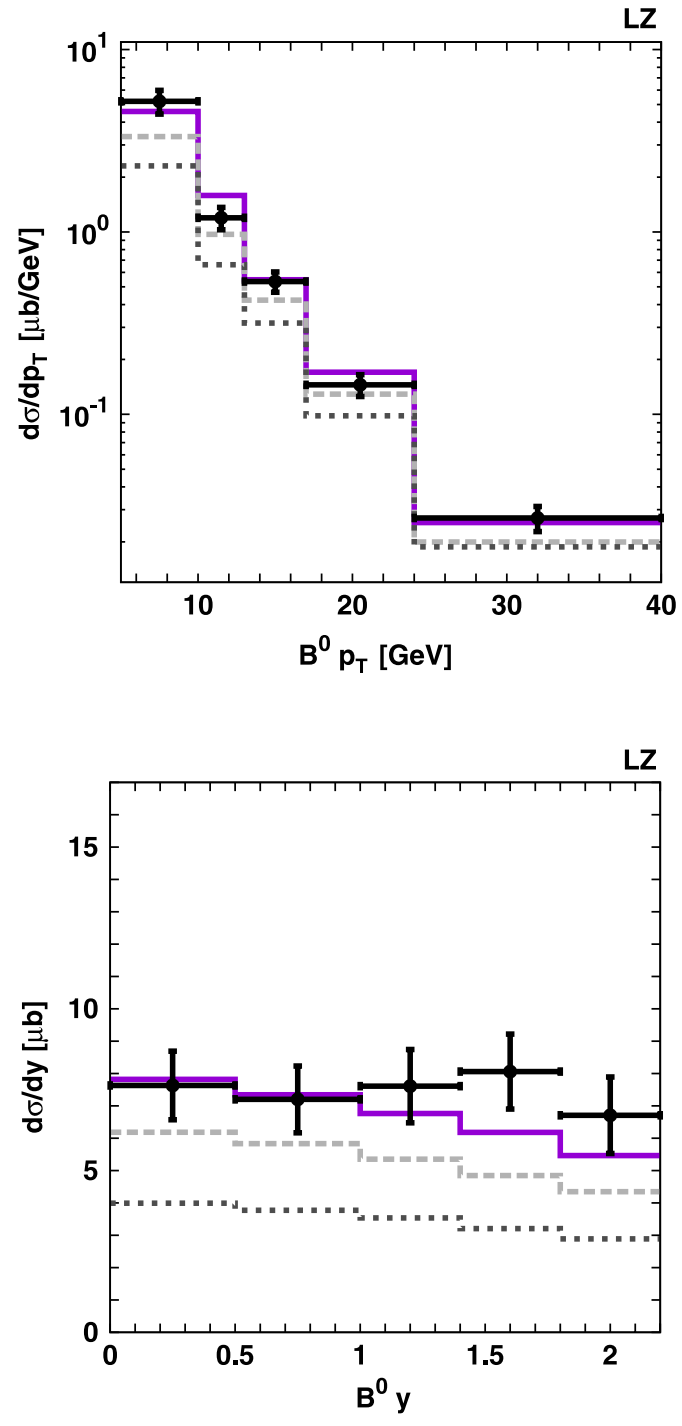

\section{NUMERICAL RESULTS}

The unintegrated gluon distributions to be used in the cross section [(1)] depend on the renormalization and factorization scales $\mu_{R}$ and $\mu_{F}$. Following [16] in the numerical calculations we set $\mu_{R}^{2}=m_{Q}^{2}+\left(\mathbf{p}_{1 T}^{2}+\mathbf{p}_{2 T}^{2}\right) / 2$, $\mu_{F}^{2}=\hat{s}+\mathbf{Q}_{T}^{2}$, where $\mathbf{Q}_{T}$ is the transverse-momentum of the initial off shell gluon pair $m_{c}=1.4 \pm 0.1 \mathrm{GeV}$, $m_{b}=4.75 \pm 0.25 \mathrm{GeV}$. We use the LO formula for the coupling $\alpha_{s}\left(\mu_{R}^{2}\right)$ with $n_{f}=4$-active quark flavors at $\Lambda_{\mathrm{QCD}}=200 \mathrm{MeV}$, such that $\alpha_{s}\left(M_{Z}^{2}\right)=0.1232$.

We are in position to present our numerical results. The CMS collaboration has measured $B^{+}$and $B^{0}$-meson cross sections in the kinematic range $p_{T}\left(B^{+}\right)>5 \mathrm{GeV}$, $\left|y\left(B^{+}\right)\right|<2.4$ [2], and $p_{T}\left(B^{0}\right)>5 \mathrm{GeV},\left|y\left(B^{0}\right)\right|<2.2$ [3],
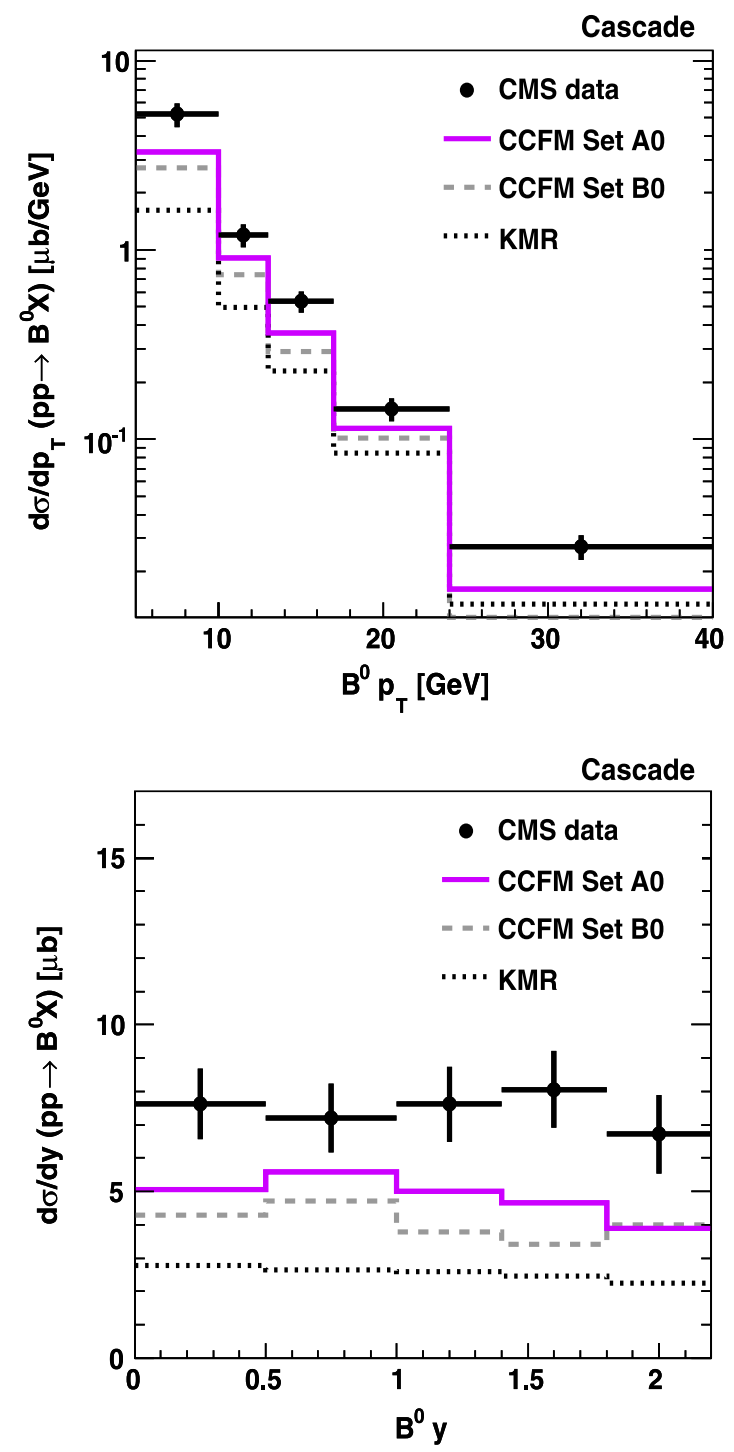

FIG. 2 (color online). The transverse-momentum and rapidity distributions of $B^{0}$-meson production at the LHC. The kinematical cuts applied are described in the text. The left histograms show the LZ numerical results while the right plots depict the CASCADE predictions. Notation of all histograms is the same as in Fig. 1. The experimental data are from CMS [3]. 
respectively. The measurements of decay-muon cross sections have been performed [4] for $p_{T}(\mu)>6 \mathrm{GeV}$ and $|\eta(\mu)|<2$.1. The LHCb collaboration have measured [1] the pseudorapidity distribution of $b$-hadrons in forward region $2<\eta\left(H_{b}\right)<6$, where $H_{b}$ is any $b$-flavored hadron. In our calculations the fragmentation of $b$ quarks into $b$ hadrons is described with the Peterson fragmentation function [25] with default value of shape parameter $\epsilon_{b}=$ 0.006 . To produce muons from $b$ quarks in the LZ calculations, we first convert $b$ quarks into $b$ hadrons and then simulate their semileptonic decay according to the standard electroweak theory. The branching fractions of $b \rightarrow B^{+}, b \rightarrow B^{0}, b \rightarrow \mu$ as well as the cascade decay $b \rightarrow c \rightarrow \mu$ are taken from [26]. The CMS collaboration has presented preliminary data [6] on the inclusive $b$-jet
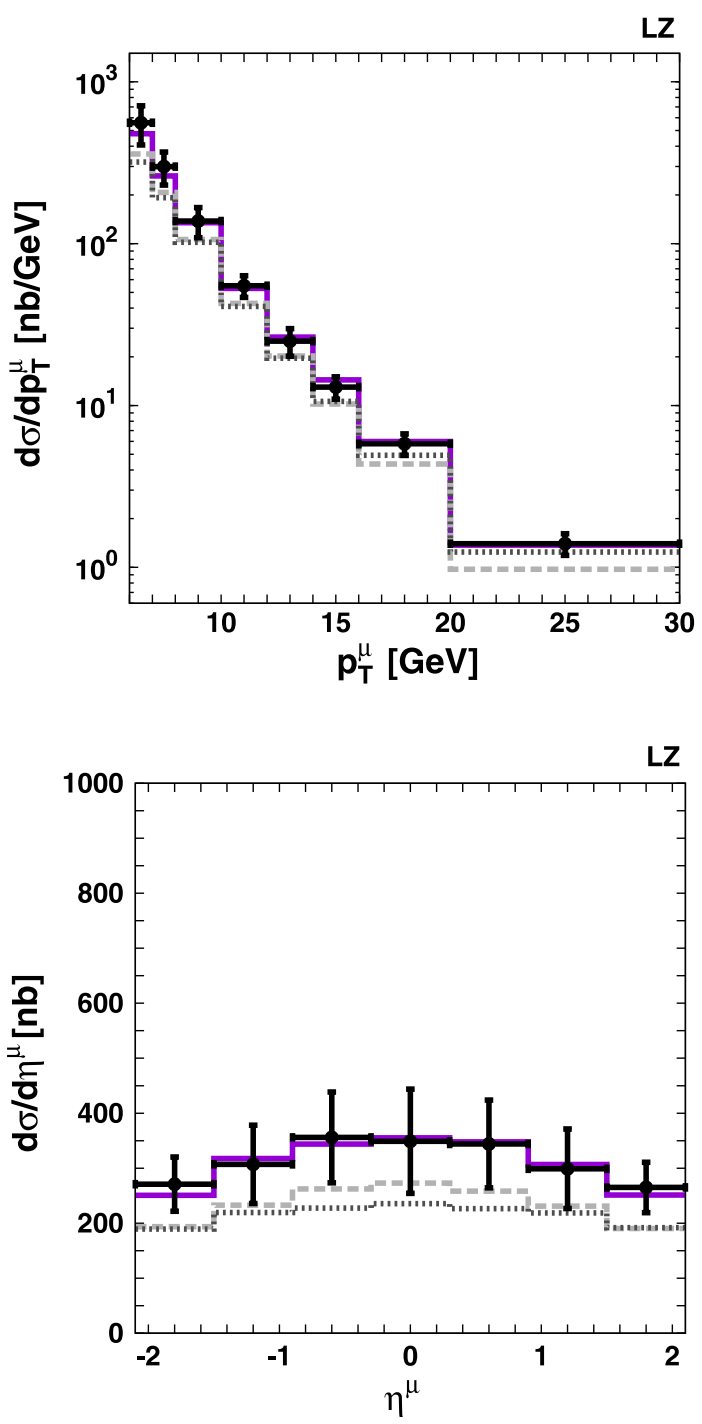

production at the $\sqrt{s}=7 \mathrm{TeV}$. The cross sections have been determined in four $b$-jet rapidity regions, namely $|y|<0.5,0.5<|y|<1,1<|y|<1.5$, and $1.5<|y|<2$. The $b$-jets in the CASCADE calculations are reconstructed with the anti- $k_{t}$ cone algorithm [27] (using the FASTJET package $[28,29]$ ) with radius $R=0.5$. Here we show only the predictions in the phase space of the CMS results [6].

The results of our calculations are shown in Figs. 1-4 in comparison with the data. We obtain a good description of the data when using the CCFM-evolved (namely, A0) gluon distribution in LZ calculations. The shape and absolute normalization of measured $b$-flavored hadron cross sections at forward rapidities are reproduced well (see Fig. 4). The KMR and CCFM B0 predictions are somewhat below the data. As it is shown in Fig. 5, in contrast with $b$
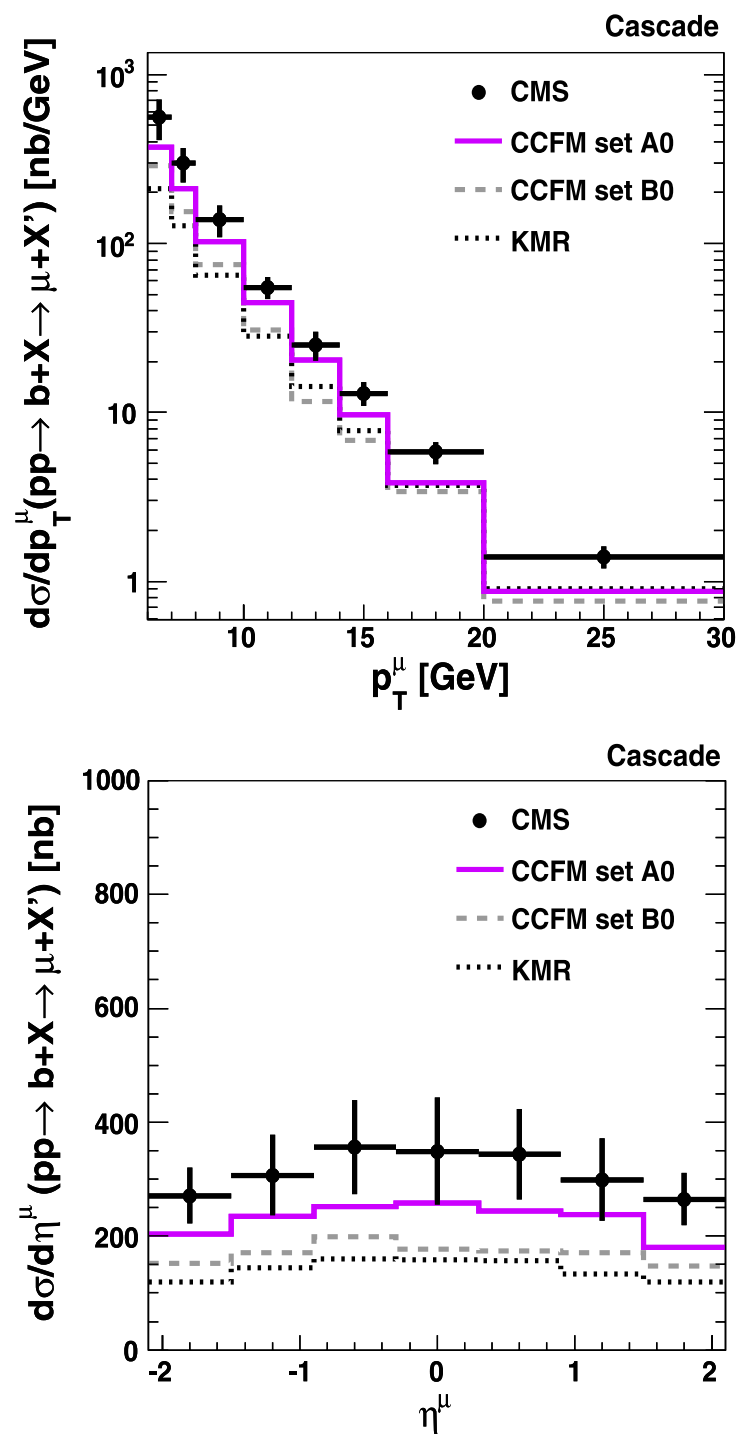

FIG. 3 (color online). The transverse-momentum and pseudorapidity distributions of muons arising from the semileptonic decays of $b$ quarks at the LHC. The kinematical cuts applied are described in the text. The left histograms show the LZ numerical results while the right plots depict the CASCADE predictions. Notation of all histograms is the same as in Fig. 1. The experimental data are from CMS [4]. 

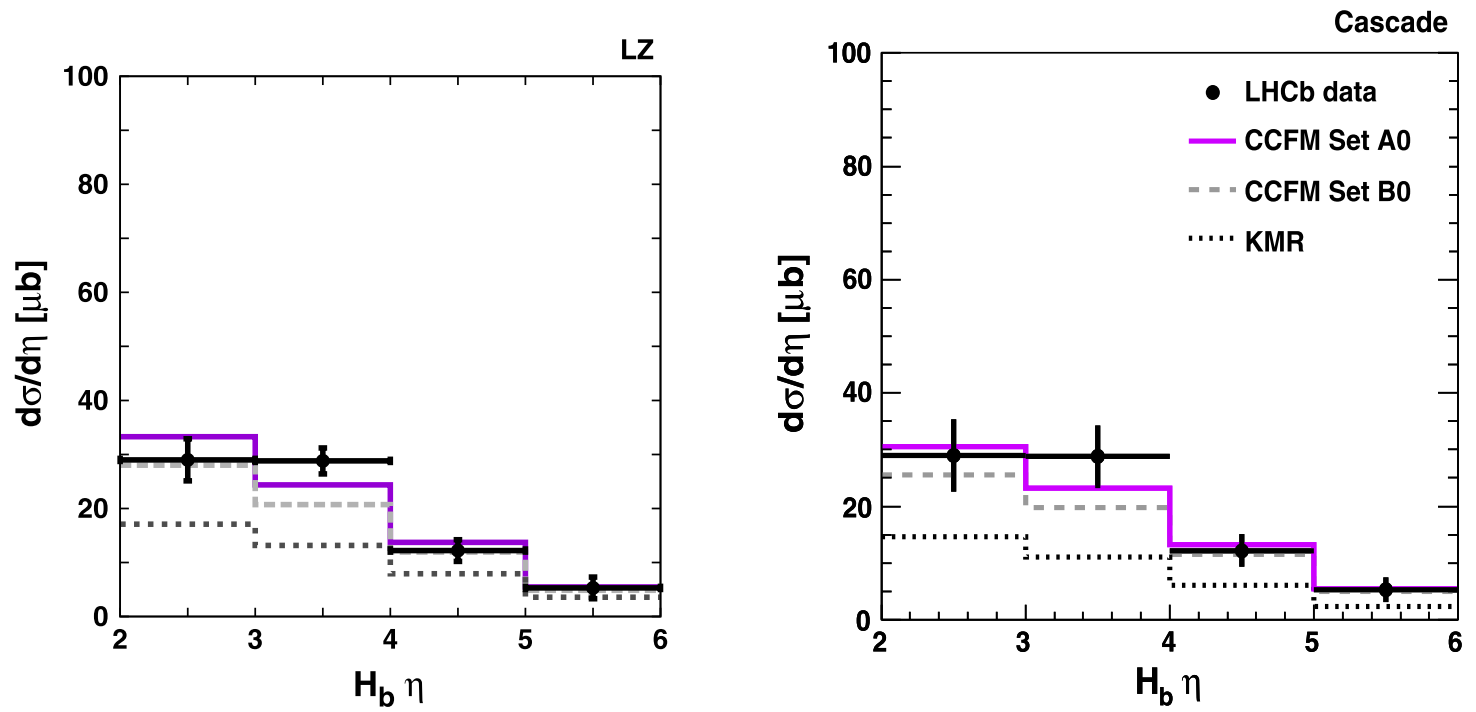

FIG. 4 (color online). The pseudorapidity distributions of $b$-flavored hadrons at the LHC. The kinematical cuts applied are described in the text. The left histogram shows the LZ numerical results while the right plot depicts the CASCADE predictions. Notation of all histograms is the same as in Fig. 1. The experimental data are from LHCb [1].
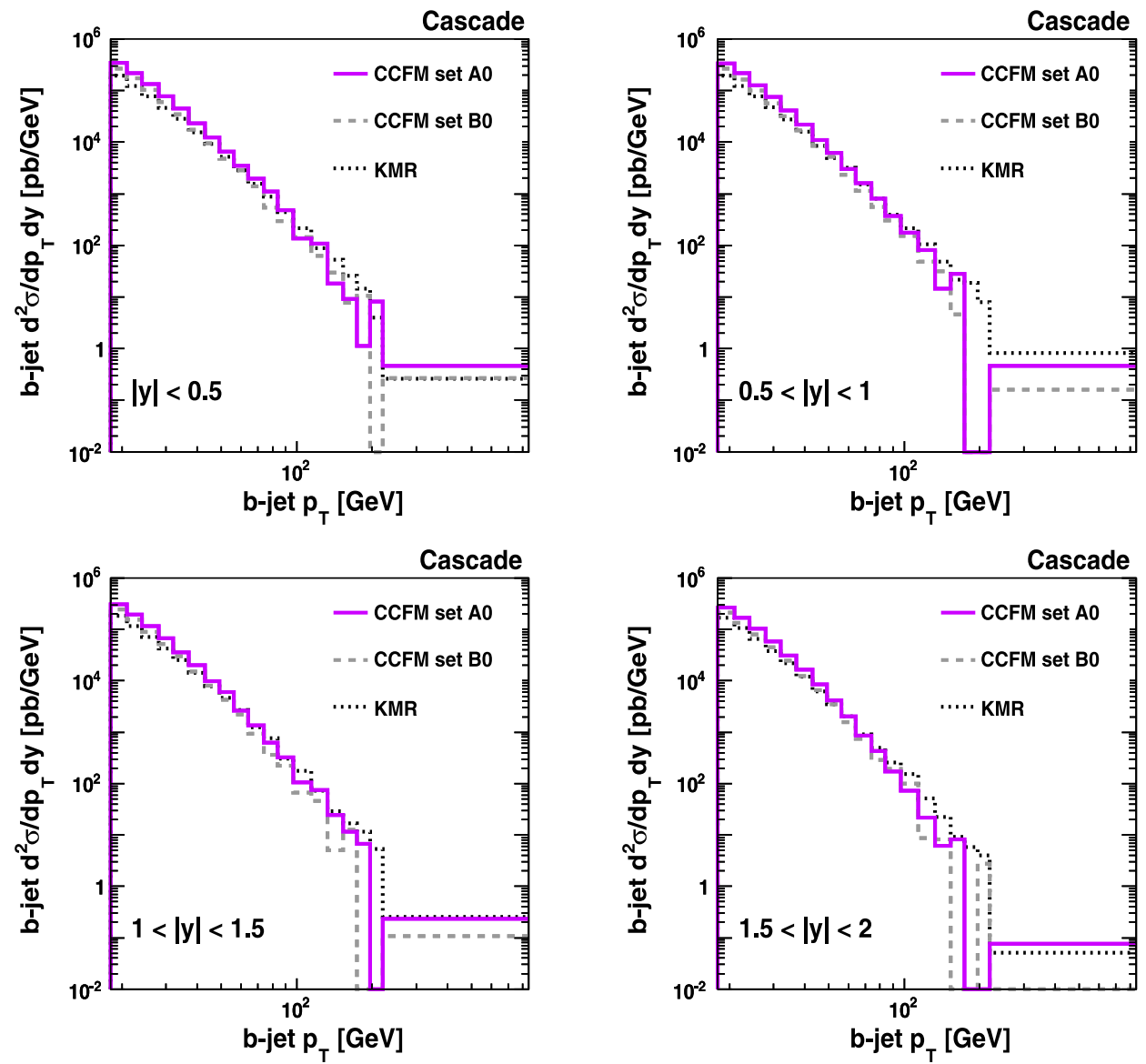

FIG. 5 (color online). The double-differential cross sections $d \sigma / d y d p_{T}$ of inclusive $b$-jet production as a function of $p_{T}$ in different $y$ regions (CASCADE predictions). Notation of all histograms is the same as in Fig. 1. 
TABLE I. The $b$-flavored hadron production cross section in $p p$ collisions at $\sqrt{s}=7 \mathrm{TeV}$.

\begin{tabular}{lccc}
\hline \hline Source & $\sigma\left(B^{+}\right)$ & $\sigma\left(B^{0}\right)$ & $\sigma(\mu)$ \\
\hline CMS data $[\mu \mathrm{b}]$ & $28.1 \pm 2.4 \pm 2.0 \pm 3.1$ & $33.2 \pm 2.5 \pm 3.5$ & $1.32 \pm 0.01 \pm 0.30 \pm 0.15$ \\
A0 (LZ/CASCADE) & $32.7 / 24.5$ & $31.4 / 24.3$ & $1.31 / 0.96$ \\
B0 (LZ/CASCADE) & $23.6 / 18.8$ & $22.5 / 20.4$ & $0.98 / 0.72$ \\
KMR (LZ/CASCADE) & $16.7 / 13.1$ & $15.8 / 12.4$ & $0.91 / 0.59$ \\
MC@ NLO [2-4] & 19.1 & 25.2 & 0.95 \\
Pythia [2-4] & 36.2 & 49.1 & 1.9 \\
\hline \hline
\end{tabular}

TABLE II. Systematic uncertainties for beauty total cross section in $p p$ collisions at $\sqrt{s}=7 \mathrm{TeV}$ obtained with CASCADE.

\begin{tabular}{lc}
\hline \hline Source & $\begin{array}{c}\sigma\left(p p \rightarrow b+X \rightarrow \mu+X^{\prime},\right. \\
\left.p_{T}^{\mu}>6 \mathrm{GeV},\left|\eta^{\mu}\right|<2.1\right)\end{array}$ \\
\hline CCFM set A0 & $0.96 \mu \mathrm{b}$ \\
CCFM set A0+ & $+13 \%$ \\
CCFM set A0- & $-2 \%$ \\
$m_{b}=5.0 \mathrm{GeV}$ & $-7 \%$ \\
$m_{b}=4.5 \mathrm{GeV}$ & $+6 \%$ \\
$\epsilon_{b}=0.003$ & $+9 \%$ \\
Total & $\pm 7 \%$ \\
\hline \hline
\end{tabular}

hadron and decay-muon cross sections, the results for inclusive $b$-jet production based on the CCFM and KMR gluons are very similar to each other. ${ }^{3}$ The CASCADE predictions tend to lie slightly below the LZ ones and are rather close to the MC@NLO calculations [7,8] (not shown). The observed difference between the LZ and CASCADE is not surprising and connected with the missing parton shower effects in the LZ evaluations. The influence of such effects is clearly demonstrated in Fig. 6, where we show separately the results of CASCADE calculations without parton shower, with only initial- and final-state and with both initial and final-state parton showers. One can see that without initial and final-state parton showers, the CASCADE predictions are very close to the LZ ones. The similar situation was pointed out previously [16] for Tevatron energies. We have checked that the LZ and CASCADE predictions coincide at parton-level.

In order to study the dependence of our predictions on the quark-to-hadron fragmentation function, we repeated our calculations with the shifted value of the Petersonshape parameter $\epsilon_{b}=0.003$, which is often used in the next-to-leading order (NLO) pQCD calculations. Additionally, we have applied the nonperturbative fragmentation functions proposed in [30-32], where the input parameters were determined in $[31,32]$ by a fit to LEP2 data. The results of our calculations are shown in Fig. 7. We find that the predicted cross sections in the considered

\footnotetext{
${ }^{3}$ Comparison of LZ predictions and preliminary CMS data on $b$-jet production are given in [18].
}

kinematic region are larger for smaller values of the parameter $\epsilon_{b}$ or if the fragmentation function from [30-32] is used. Thus, the LHC data lie within the band of theoretical uncertainties.

The visible cross sections of $b$-flavored hadrons and $b$-decay muons are listed in Table I in comparison with the CMS data [2-4]. In Table II the systematic uncertainties of our calculations are summarized. To estimate the uncertainty coming from the renormalization scale $\mu_{R}$, we used the CCFM set $\mathrm{AO}+$ and $\mathrm{A} 0$ - instead of the default density function A0. These two sets represent a variation of the scale used in $\alpha_{s}$ in the off shell matrix element. The A $0+$ stands for a variation of $2 \mu_{R}$, while set $\mathrm{A} 0-$ reflects $\mu_{R} / 2$. We observe a deviation of roughly $13 \%$ for set $\mathrm{A} 0+$. The uncertainty coming from set $\mathrm{A} 0-$ is generally smaller and negative. The dependence on the $b$-quark mass is investigated by a variation of $b$-quark mass of $m_{b}=$ $4.75 \mathrm{GeV}$ by $\pm 0.25 \mathrm{GeV}$. The calculated that $b$-quark cross sections vary by $\sim \pm 6 \%$.

Now we turn to the investigation of the angular correlations between the produced $b$ hadrons. As it was pointed out in [14-16], such observables are very sensitive to the details of the noncollinear gluon evolution. The CMS collaboration [5] has measured the $b$-flavored hadron pair production as a function of the angular separation $\Delta \phi$ between the two reconstructed $b$ hadrons and variable $\Delta R=\sqrt{(\Delta \eta)^{2}+(\Delta \phi)^{2}}$ for three different event scales characterized by the leading jet transverse-momentum $p_{T}$, namely, $p_{T}>56 \mathrm{GeV}, p_{T}>84 \mathrm{GeV}$, and $p_{T}>$ $120 \mathrm{GeV}$. The kinematic range for the measurements is defined by the requirements $p_{T}\left(H_{b}\right)>15 \mathrm{GeV}$ and $\left|\eta\left(H_{b}\right)\right|<2$ for both $b$-flavored hadrons. The leading jet is required to be within $|\eta|<3$.0. Our predictions for $\Delta \phi$ and $\Delta R$ distributions are shown in Figs. 8 and 9. One can see that none of the calculations fully describes the LHC data and, therefore, there is a room for further studies. Note that the predicted shapes of $\Delta \phi$ and $\Delta R$ distributions are very different for different unintegrated gluon densities used, as was expected. This is in contrast to the cross sections as a function of transverse momenta or rapidities, where all gluon distributions gave similar behavior. Note also that the measured cross sections at small $\Delta \phi$ or $\Delta R$ are significant. Moreover, they exceed the ones observed at large angular separation where the two $b$-flavored hadrons 

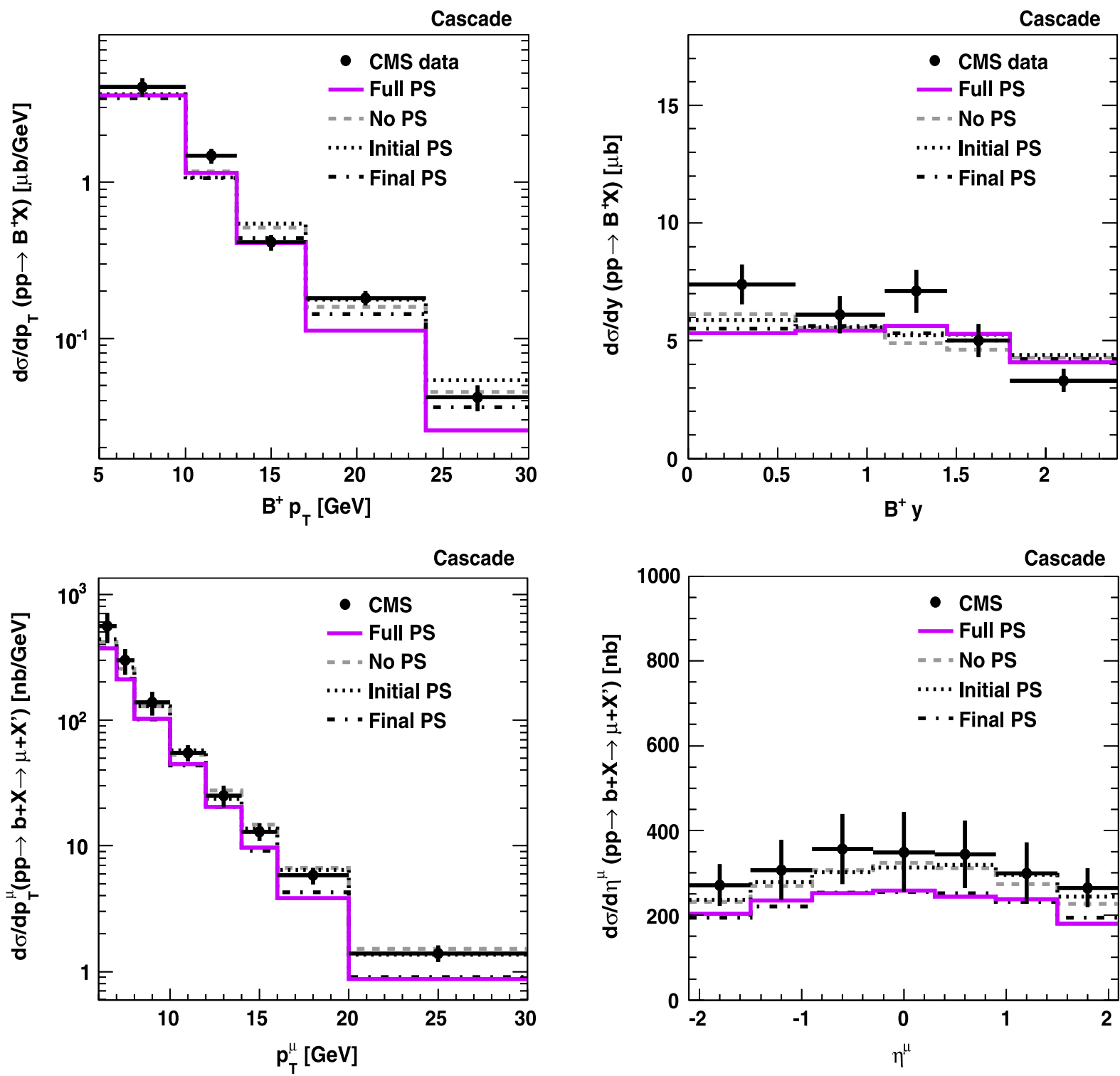

FIG. 6 (color online). Parton shower effects in the pseudorapidity and transverse-momentum distributions of the $b$-quark decay muons. The four lines represent full parton shower (solid line), no parton shower (dashed line), initial-state parton shower (dotted line) and final-state parton shower (dashed, dotted line). The experimental data are from CMS [2,4].

are emitted in opposite directions. This behavior is reproduced by the KMR gluon density only due to different tail at large $\mathbf{k}_{T}$ compared to the CCFM-evolved gluon distributions. ${ }^{4}$ In general this difference is associated with two different approaches: the "exclusive" approach to unintegrated parton distribution functions (uPDF) [20] and the "inclusive" one [34] based on connecting the uPDFs to the ordinary PDFs via resummed renormalization-group equations, which resulted in the uPDFs of [22]. These two approaches coincide at the level of small $x$ LO parton

\footnotetext{
${ }^{4} \mathrm{~A}$ detailed comparison of KMR and CCFM gluon distributions can be found in [33].
}

evolution but give rise to different subleading corrections, which may well be important in detailed final-state observables, such as particle correlations in small $\Delta \phi$ and $\Delta R$ region.

The role of nonzero gluon transverse-momentum $\mathbf{k}_{T}$ is also clearly illustrated in Fig. 10. Here, the solid histograms correspond to the results obtained according to the master formula [(1)] and the dotted histograms are obtained by using the same formula and without virtualities of the incoming gluons in partonic amplitude. In the last case, an additional requirement $\mathbf{k}_{1,2 T}^{2}<\mu_{R}^{2}$ is applied. One can see that the gluon transverse-momentum $\mathbf{k}_{T}$ (both in the hard matrix element and in the gluon distribution 

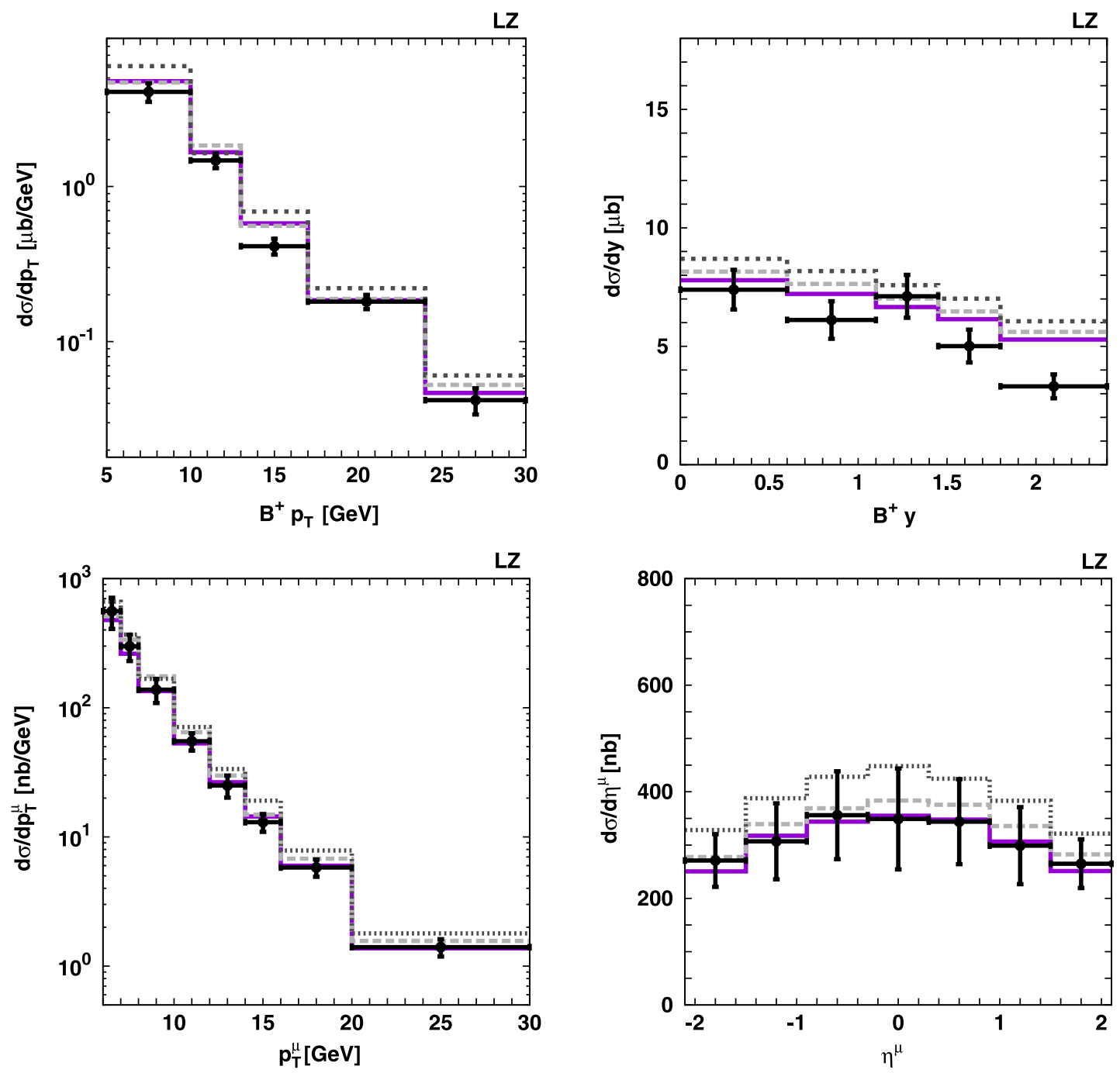

FIG. 7 (color online). The dependence of our predictions on the fragmentation scheme (LZ calculations). The solid, dashed and dotted histograms correspond to the results obtained using the Peterson fragmentation function with $\epsilon_{b}=0.006, \epsilon_{b}=0.003$, and the nonperturbative fragmentation functions from [23-25], respectively. The CCFM-evolved (A0) gluon density is applied. The experimental data are from CMS $[2,4]$.

functions) is important for description of the LHC data at low $\Delta \phi$ or $\Delta R$. The effect observed here seems to be general and goes beyond the $b$-quark observable considered. For example, a similar effect was observed in the study of angular correlations of jets in deep inelastic scattering process $[33,35]$. We see in Figs. 8 and 9 that even using transverse-momentum-dependent parton distributions, the shape of the angular correlations requires finite- $\mathbf{k}_{T}$ terms in the short-distance matrix elements.

\section{CONCLUSIONS}

In this paper, we analyzed the first data on the beauty production in $p p$ collisions at the LHC taken by the CMS and $\mathrm{LHCb}$ collaborations. Our consideration is based on the $k_{T}$-factorization approach supplemented with the
CCFM-evolved unintegrated gluon densities in a proton. The analysis covers the total and differential cross sections of $b$-flavored hadrons and muons originating from the semileptonic decays of beauty quarks as well as the double-differential cross sections of inclusive $b$-jet production. Using the full hadron-level Monte Carlo generator CASCADE, we investigated the effects coming from the parton showers in initial and final states. Different sources of theoretical uncertainties have been studied.

Our LZ predictions with the default set of parameters agree with the data on the transverse-momentum and pseudorapidity distributions of $b$-flavored hadrons or $b$-quark decay muons. The CASCADE predictions tend to slightly underestimate the data at central rapidities, but the data points still lie within the band of theoretical 

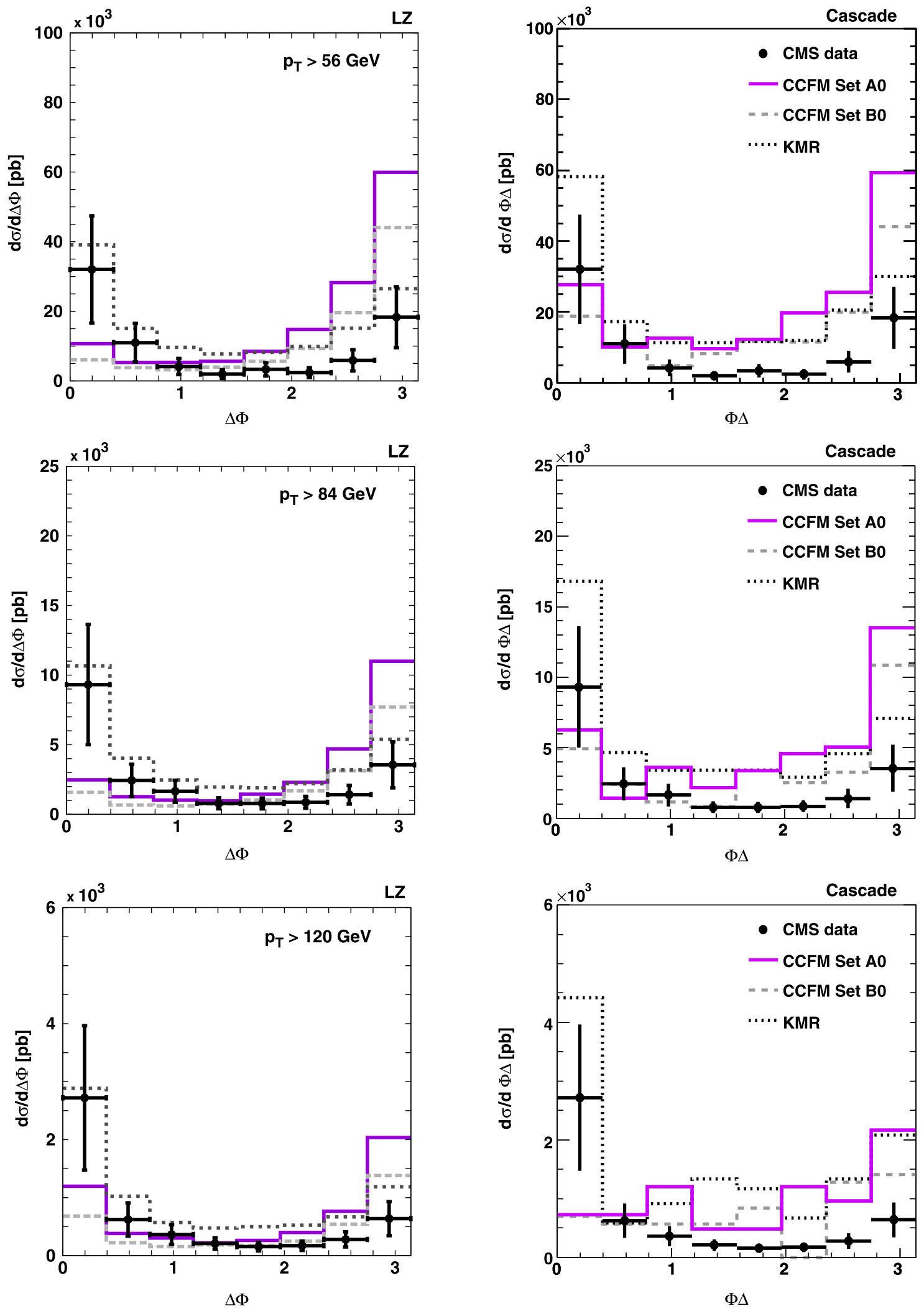

FIG. 8 (color online). The distributions in azimuthal angle difference between the produced $b$-flavored hadrons at the LHC. The first column shows the LZ numerical results while the second one depicts the CASCADE predictions. The kinematical cuts applied are described in the text. Notation of all histograms is the same as in Fig. 1. The experimental data are from CMS [5]. 

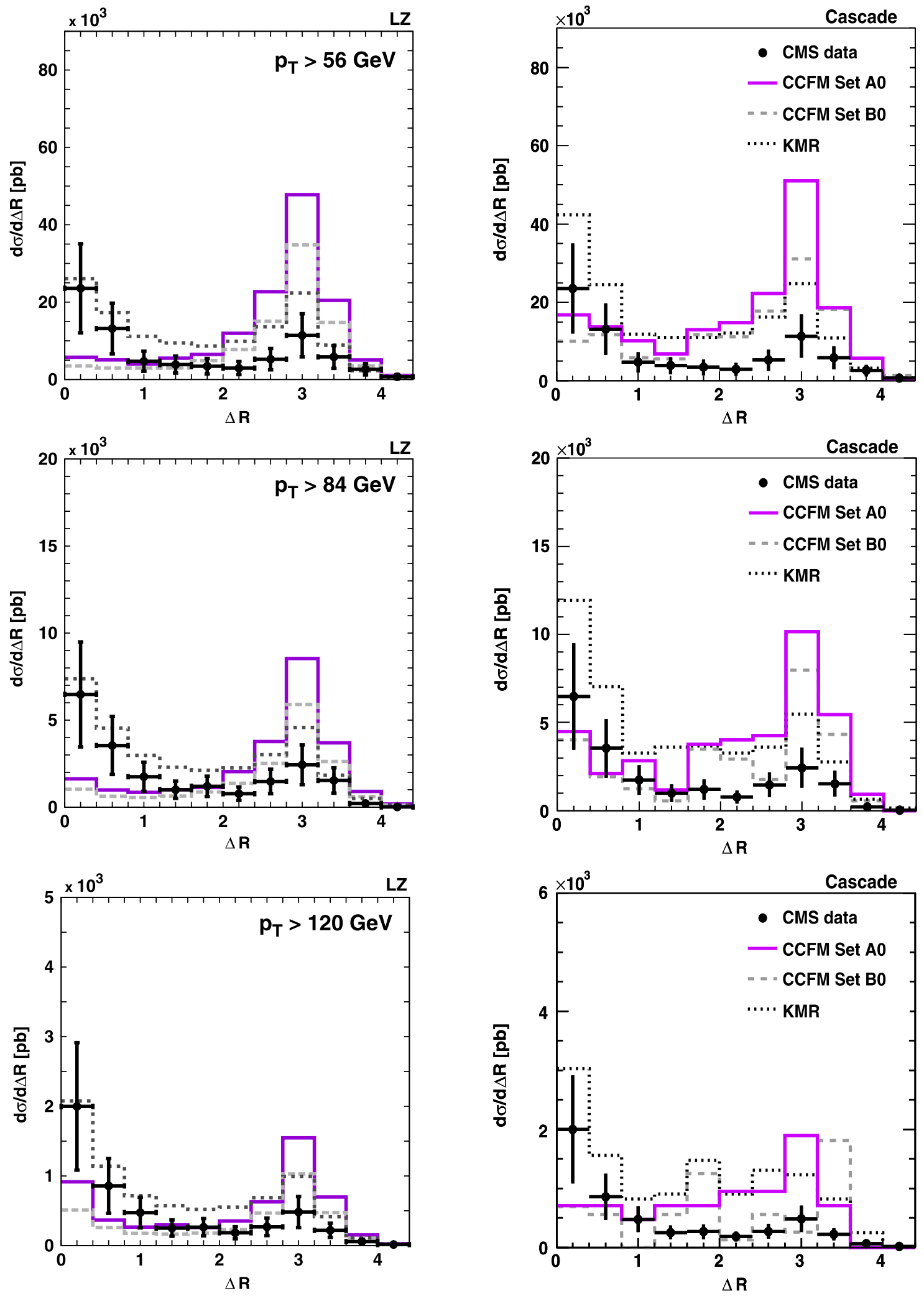

FIG. 9 (color online). The distributions in $\Delta R$ in the $b$-flavored hadron production at the LHC. The first column shows the LZ numerical results while the second one depicts the CASCADE predictions. The kinematical cuts applied are described in the text. Notation of all histograms is the same as in Fig. 1. The experimental data are from CMS [5]. 

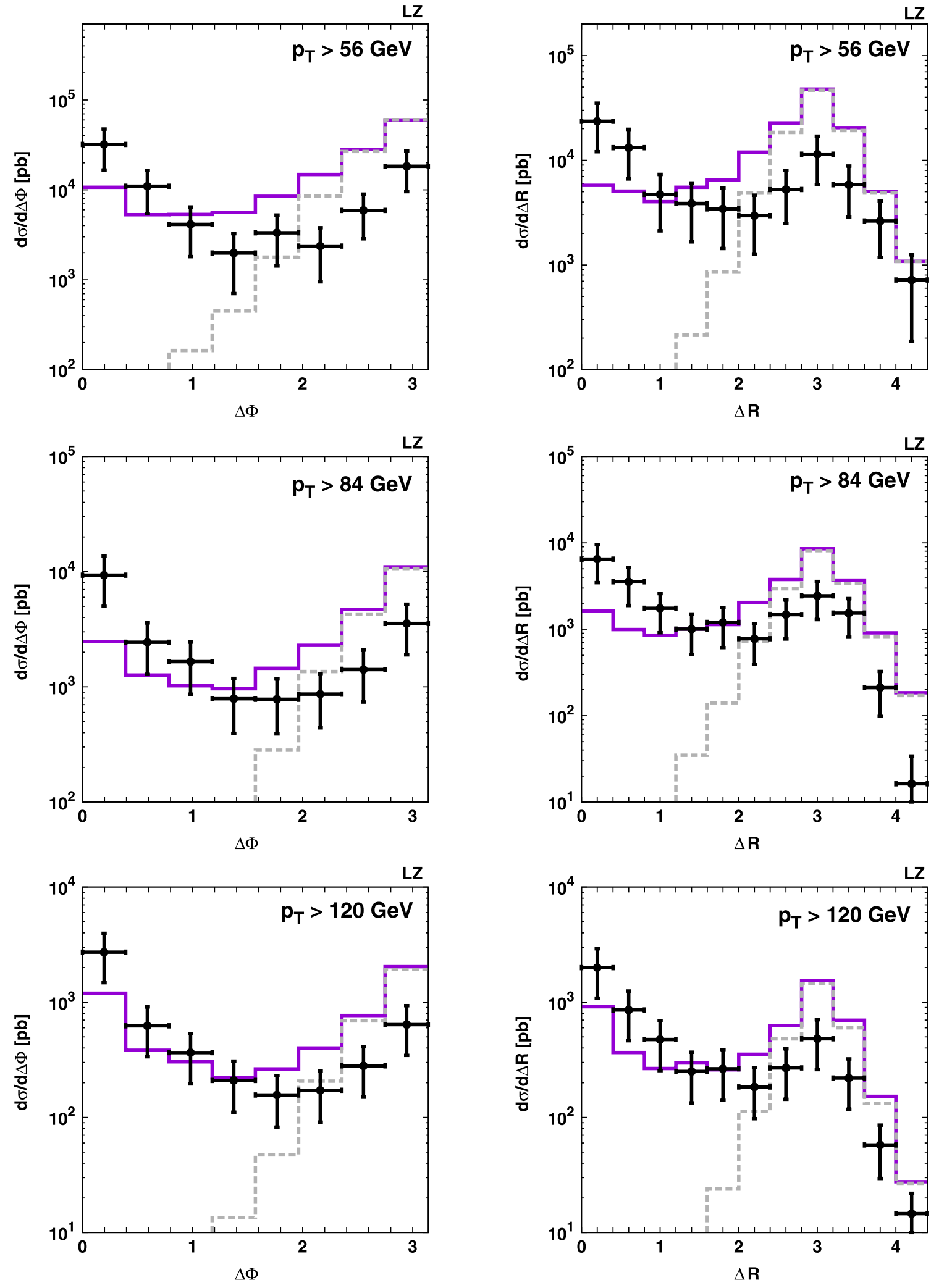

FIG. 10 (color online). Importance of nonzero transverse-momentum of incoming gluons in open $b$-quark production at the LHC. The solid histograms correspond to the results obtained according to the master formula [(1)]. The dotted histograms are obtained by using the same formula, but now we switch off the virtualities of both incoming gluons in partonic amplitude and apply an additional requirement $\mathbf{k}_{1,2 T}^{2}<\mu_{R}^{2}$. We have used here the CCFM A0 gluon for illustration. The experimental data are from CMS [5]. 
uncertainties. In this case, the overall description of the data is at a similar level of agreement as in the framework of NLO collinear QCD factorization. Special attention was put on the analysis of specific angular correlations between the produced $b$-flavored hadrons measured by the CMS collaboration. The description of $\Delta \phi$ and $\Delta R$ distributions in the framework of the $k_{T}$-factorization require further studies.

\section{ACKNOWLEDGMENTS}

We are grateful to comments from V. Chiochia, G. Dissertori, W. Erdmann, A. Rizzi, V. Zhukov, and
S. Baranov. The authors are very grateful to Deutsches Elektronen-Synchrotron (DESY) Directorate for the support in the framework of the Moscow-DESY project on Monte-Carlo implementation for Hadron Electron Ring Accelerator-Large Hadron Collider (LHC). A. V. L. was supported in part by the president of Russian Federation, Grant No. MK-3977.2011.2. Also, this research was supported by the Federal Agency for Science and Innovation of Russian Federation Grant No. NS-4142.2010.2, Federal Agency for Science and Innovation state Contract No. 02.740.11.0244, Russian Federation for Basic Research Grant No. 11-02-01454-a, and the RMES (grant the Scientific Research on High Energy Physics).
[1] LHCb Collaboration, Phys. Lett. B 694, 209 (2010).

[2] CMS Collaboration, Phys. Rev. Lett. 106, 112001 (2011).

[3] CMS Collaboration, Phys. Rev. Lett. 106, 252001 (2011).

[4] CMS Collaboration, J. High Energy Phys. 03 (2011) 090.

[5] CMS Collaboration, J. High Energy Phys. 03 (2011) 136.

[6] CMS Collaboration, Report No. CMS-PAS-BPH-10-009, https://cdsweb.cern.ch/record/1280454?ln=en.V. Chiochia, Nucl. Phys. A855, 436 (2011).

[7] S. Frixione and B. R. Webber, J. High Energy Phys. 06 (2002) 029.

[8] S. Frixione, P. Nason, and B. R. Webber, J. High Energy Phys. 08 (2003) 007.

[9] L. V. Gribov, E. M. Levin, and M. G. Ryskin, Phys. Rep. 100, 1 (1983); E. M. Levin, M. G. Ryskin, Yu. M. Shabelsky, and A. G. Shuvaev, Sov. J. Nucl. Phys. 53, 657 (1991); S. Catani, M. Ciafoloni, and F. Hautmann, Phys. Lett. B 242, 97 (1990); Nucl. Phys. B366, 135 (1991);J. C. Collins and R. K. Ellis, Nucl. Phys. B360, 3 (1991).

[10] S. P. Baranov and M. Smizanska, Phys. Rev. D 62, 014012 (2000).

[11] Ph. Hägler, R. Kirschner, A. Schäfer, L. Szymanowski, and O. V. Teryaev, Phys. Rev. D 62, 071502 (2000).

[12] M. G. Ryskin, Yu. M. Shabelski, and A. G. Shuvaev, Phys. At. Nucl. 64, 1995 (2001); Yu. M. Shabelski and A. G. Shuvaev, Phys. At. Nucl. 69, 314 (2006).

[13] N.P. Zotov, A. V. Lipatov, and V. A. Saleev, Phys. At. Nucl. 66, 755 (2003).

[14] S. P. Baranov, N. P. Zotov, and A. V. Lipatov, Phys. At. Nucl. 67, 837 (2004).

[15] A. V. Lipatov, L. Lönnblad, and N.P. Zotov, J. High Energy Phys. 01 (2004) 010.

[16] H. Jung, M. Krämer, A. V. Lipatov, and N. P. Zotov, J. High Energy Phys. 01 (2011) 085.

[17] H. Jung, Comput. Phys. Commun. 143, 100 (2002); H. Jung et al., Eur. Phys. J. C 70, 1237 (2010).

[18] H. Jung, M. Kraemer, A. V. Lipatov, and N.P. Zotov, arXiv:1107.4328.
[19] E. A. Kuraev, L. N. Lipatov, and V.S. Fadin, Sov. Phys. JETP 44, 443 (1976); E. A. Kuraev, L. N. Lipatov, and V. S. Fadin, Sov. Phys. JETP 45, 199 (1977); I. I. Balitsky and L. N. Lipatov, Sov. J. Nucl. Phys. 28, 822 (1978).

[20] M. Ciafaloni, Nucl. Phys. B296, 49 (1988); S. Catani, F. Fiorani, and G. Marchesini, Phys. Lett. B 234, 339 (1990); Nucl. Phys. B336, 18 (1990); G. Marchesini, Nucl. Phys. B445, 49 (1995).

[21] H. Jung, in Proceedings of the DIS' 2004 (International Workshop on Deep Inelastic Scattering, Strbske Pleso, Slovakia, 2004).

[22] M. A. Kimber, A. D. Martin, and M. G. Ryskin, Phys. Rev. D 63, 114027 (2001); G. Watt, A. D. Martin, and M. G. Ryskin, Eur. Phys. J. C 31, 73 (2003).

[23] A. D. Martin, W. J. Stirling, R. S. Thorne, and G. Watt, Eur. Phys. J. C 63, 189 (2009).

[24] A. D. Martin, R. G. Roberts, W. J. Stirling, and R. S. Thorne, Eur. Phys. J. C 14, 133 (2000).

[25] C. Peterson, D. Schlatter, I. Schmitt, and P. Zerwas, Phys. Rev. D 27, 105 (1983).

[26] K. Hagiwara et al. (PDG Collaboration), Phys. Rev. D 66, 010001 (2002).

[27] M. Cacciari, G. P. Salam, and G. Soyez, J. High Energy Phys. 04 (2008) 063.

[28] M. Cacciari and G. P. Salam, Phys. Lett. B 641, 57 (2006).

[29] M. Cacciari and G. Soyez, http://fastjet.fr/.

[30] M. Cacciari and P. Nason, Phys. Rev. Lett. 89, 122003 (2002); M. Cacciari, S. Frixione, M. L. Mangano, P. Nason, and G. Ridolfi, J. High Energy Phys. 07 (2004) 033.

[31] M. Cacciari and P. Nason, J. High Energy Phys. 09 (2003) 006.

[32] E. Braaten, K.-M. Cheng, S. Fleming, and T.C. Yuan, Phys. Rev. D 51, 4819 (1995).

[33] B. Andersson et al. (Small- $x$ Collaboration), Eur. Phys. J. C 25, 77 (2002); J. Andersen et al. (Small-x Collaboration), Eur. Phys. J. C 35, 67 (2004); 48, 53 (2006).

[34] S. Catani, M. Ciafoloni, and F. Hautmann, Phys. Lett. B 307, 147 (1993).

[35] F. Hautmann and H. Jung, J. High Energy Phys. 10 (2008) 113. 